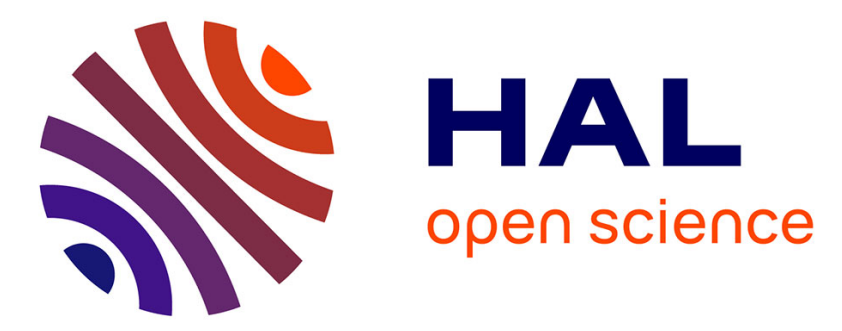

\title{
The Context-Freeness Problem Is coNP-Complete for Flat Counter Systems
}

\author{
Jérôme Leroux, Vincent Penelle, Grégoire Sutre
}

\section{To cite this version:}

Jérôme Leroux, Vincent Penelle, Grégoire Sutre. The Context-Freeness Problem Is coNP-Complete for Flat Counter Systems. ATVA'14, Nov 2014, Sydney, Australia. pp.248 - 263, 10.1007/978-3-31911936-6_19 . hal-01084819

\section{HAL Id: hal-01084819 https://hal.science/hal-01084819}

Submitted on 20 Nov 2014

HAL is a multi-disciplinary open access archive for the deposit and dissemination of scientific research documents, whether they are published or not. The documents may come from teaching and research institutions in France or abroad, or from public or private research centers.
L'archive ouverte pluridisciplinaire HAL, est destinée au dépôt et à la diffusion de documents scientifiques de niveau recherche, publiés ou non, émanant des établissements d'enseignement et de recherche français ou étrangers, des laboratoires publics ou privés. 


\title{
The Context-Freeness Problem is coNP-complete for Flat Counter Systems ${ }^{\star}$
}

\author{
Jérôme Leroux ${ }^{1}$, Vincent Penelle ${ }^{2}$, and Grégoire Sutre ${ }^{1}$ \\ 1 Univ. Bordeaux \& CNRS, LaBRI, UMR 5800, Talence, France \\ 2 Univ. Paris Est \& CNRS, LIGM, UMR 8049, Marne-la-Vallée, France
}

\begin{abstract}
Bounded languages have recently proved to be an important class of languages for the analysis of Turing-powerful models. For instance, bounded context-free languages are used to under-approximate the behaviors of recursive programs. Ginsburg and Spanier have shown in 1966 that a bounded language $L \subseteq a_{1}^{*} \cdots a_{d}^{*}$ is context-free if, and only if, its Parikh image is a stratifiable semilinear set. However, the question whether a semilinear set is stratifiable, hereafter called the stratifiability problem, was left open, and remains so. In this paper, we give a partial answer to this problem. We focus on semilinear sets that are given as finite systems of linear inequalities, and we show that stratifiability is coNP-complete in this case. Then, we apply our techniques to the context-freeness problem for flat counter systems, that asks whether the trace language of a counter system intersected with a bounded regular language is context-free. As main result of the paper, we show that this problem is coNP-complete.
\end{abstract}

\section{Introduction}

The class of bounded languages was introduced in 1964 by Ginsburg and Spanier to study context-free languages [11]. Nowadays, this class plays an important role in the analysis of Turing-powerful models. Recall that a language is bounded if it is contained in $\sigma_{1}^{*} \cdots \sigma_{d}^{*}$ for some words $\sigma_{1}, \ldots, \sigma_{d}$. The restriction of a model to behaviors contained in a bounded language produces a so-called flat model that is often amenable to automatic verification. Indeed, the reachability sets of such restrictions are usually computable through acceleration techniques $[3,4,8,1,10,2,5]$. Following the same approach, bounded languages have recently been used for the analysis of recursive concurrent systems, and more generally as a way to unify various recent and promising "bounded verification" techniques [9].

In these applications, the class of semilinear bounded languages is central. Recall that a semilinear set is a finite union of sets of the form $\boldsymbol{b}+\mathbb{N} \boldsymbol{p}_{1}+\cdots+\mathbb{N} \boldsymbol{p}_{k}$ where $\boldsymbol{b}, \boldsymbol{p}_{1}, \ldots, \boldsymbol{p}_{k}$ are vectors in $\mathbb{N}^{d}$. Semilinear sets coincide with the sets definable in Presburger arithmetic [13]. A semilinear bounded language is a language of the form $\left\{\sigma_{1}^{n_{1}} \cdots \sigma_{d}^{n_{d}} \mid\left(n_{1}, \ldots, n_{d}\right) \in \boldsymbol{S}\right\}$ where $\sigma_{1}, \ldots, \sigma_{d}$ are words and $\boldsymbol{S}$ is a semilinear set. The class of semilinear bounded languages admits several characterizations through language acceptors $[6,16,9]$. From a language-theoretic

\footnotetext{
* This work was supported by the ANR project REACHARD (ANR-11-BS02-001).
} 
viewpoint, semilinear bounded languages are incomparable with context-free languages. Indeed, the language $\left\{a^{n} b^{n} c^{n} \mid n \in \mathbb{N}\right\}$ is well-known to be noncontext-free, and, conversely, the language $\{a, b\}^{*}$ is not a bounded language. However, bounded context-free languages are semilinear bounded languages, by Parikh's theorem and closure under inverse morphism of context-free languages.

Ginsburg and Spanier have established in $[13,14]$ a characterization of bounded context-free languages in terms of semilinear sets satisfying a "stratification" requirement. We call such semilinear sets stratifiable. The existence of a decision procedure for determining whether a given semilinear set is stratifiable was left open in [13,14], and has remained open since then. Rephrased in terms of languages, this decision problem is equivalent to the question whether a given semilinear bounded language is context-free. The latter problem is known to be decidable for some subclasses of semilinear bounded languages, with a notable example being the trace languages of flat Petri nets. In fact, the context-freeness problem is decidable for trace languages of arbitrary Petri nets [22,18], and was recently shown to be EXPSPACE-complete for them [19].

Contributions. In this paper, we provide a partial answer to the question whether a given semilinear set is stratifiable, hereafter called the stratifiability problem. We focus on semilinear sets that are integral polyhedra, in other words, that are given as finite systems of linear inequalities. Our contributions are twofold.

As main technical result of the paper, we show that the stratifiability problem for integral polyhedra is coNP-complete. The proof is decomposed in two steps. First, we reduce the stratifiability of an integral polyhedron $\left\{\boldsymbol{x} \in \mathbb{N}^{d} \mid \boldsymbol{A x} \geq \boldsymbol{b}\right\}$ to a stratification-like property, called nestedness, that only involves the matrix $\boldsymbol{A}$. We then provide a criterion for nestedness, and show how to express this criterion by a polynomial-size quantifier-free formula in the first-order theory of the rational numbers with addition and order. This way, we obtain that the stratifiability problem for integral polyhedra is solvable in coNP. The proof of coNP-hardness is by reduction from the emptiness problem for integral polyhedra.

Building on this result, we then investigate the context-freeness problem for flat counter systems, that asks whether the trace language of a counter system intersected with a bounded regular language is context-free. In our setting, counter systems are a generalization of Petri nets where transitions are guarded by integral polyhedra. Such guards can express zero tests, so counter systems are Turing-powerful since they subsume Minsky machines. By exploiting the restriction to bounded languages required by flatness, we show that the contextfreeness problem for flat counter systems is coNP-complete, and remains so for flat Petri nets.

Related Work. The class of semilinear bounded languages was recently characterized through various language acceptors, namely, Parikh automata [6], reversal-bounded counter machines [16], and multi-head pushdown automata [9]. The class of semilinear sets was shown in [20] to coincide with the finite intersections of stratifiable semilinear sets. It follows that the class of bounded 
context-free languages is a generating class for the semilinear bounded languages. In [17], the stratifiability problem is shown to be equivalent to the existence of a 0 -synchronized $n$-tape pushdown automaton equivalent to a given $n$-tape finite-state automaton whose language is contained in $a_{1}^{*} \times \cdots \times a_{n}^{*}$. In a recent paper [18], we proved that the trace language of a Petri net is context-free if, and only if, it has a context-free intersection with every bounded regular language. Building on this characterization, we then established in [19] that the contextfreeness problem for Petri nets is EXPSPACE-complete, but the complexity was left open for the subcase of flat Petri nets. Here, we show that the context-freeness problem for flat Petri nets is coNP-complete. Related to our work is the question whether a given model is a posteriori flat, in other words, whether the set of all its behaviors is a bounded language. This question is shown in [7] to be decidable for the class of complete and deterministic well-structured transition systems.

Outline. The paper is organized as follows. Preliminary notations are given in Section 2. We recall the definition of stratifiable semilinear sets in Section 3. The stratifiability problem for integral polyhedra is shown to be decidable in Section 4, and it is proved to be coNP-complete in Section 5. We then address the context-freeness problem for flat counter systems, and show in Section 6 that it is coNP-complete. Section 7 concludes the paper with directions for future work.

\section{Preliminaries}

We let $\mathbb{N}, \mathbb{Z}$ and $\mathbb{Q}$ denote the usual sets of nonnegative integers, integers and rational numbers, respectively. We write $\mathbb{N}_{1}$ for the set of positive integers and $\mathbb{Q}_{\geq 0}$ for the set of nonnegative rational numbers. Vectors (of rational numbers), sets of vectors and matrices are typeset in bold face. The $i$ th component of a vector $\boldsymbol{v}$ is written $\boldsymbol{v}(i)$. The support of a vector $\boldsymbol{v}$, written $\operatorname{supp}(\boldsymbol{v})$, is the set of indices $i$ such that $\boldsymbol{v}(i) \neq 0$. We let $\boldsymbol{e}_{i}$ denote the $i$ th unit vector, defined by $\boldsymbol{e}_{i}(i)=1$ and $\boldsymbol{e}_{i}(j)=0$ for all indices $j \neq i$.

A partial-order on a set $S$ is a binary relation $\preceq$ on $S$ that is reflexive, antisymmetric and transitive. As usual, we write $s \prec t$ when $s \preceq t$ and $t \npreceq s$. A well-partial-order on $S$ is a partial-order $\preceq$ on $S$ such that every infinite sequence $s_{0}, s_{1}, s_{2}, \ldots$ in $S$ contains an increasing pair $s_{i} \preceq s_{j}$ with $i<j$.

\section{Stratifiable Semilinear Sets}

Building on earlier work with Spanier [13], Ginsburg provides, in his book [14], a characterization of bounded context-free languages in terms of semilinear sets satisfying a "stratification" requirement. We call such semilinear sets stratifiable. The existence of a decision procedure for determining whether a given semilinear set is stratifiable was left open in [13,14], and has remained open since then. We provide a partial answer to this problem in Sections 4 and 5. Before that, we 
recall in this section the definition of stratifiable semilinear sets, and how they can be used to characterize bounded context-free languages.

Given a finite set $\boldsymbol{P}=\left\{\boldsymbol{p}_{1}, \ldots, \boldsymbol{p}_{k}\right\}$ of vectors in $\mathbb{N}^{d}$, we let $\boldsymbol{P}^{\star}$ denote the set of finite sums of vectors in $\boldsymbol{P}$, i.e., $\boldsymbol{P}^{\star}=\mathbb{N} \boldsymbol{p}_{1}+\cdots+\mathbb{N} \boldsymbol{p}_{k}$. A linear set is a set of the form $\boldsymbol{b}+\boldsymbol{P}^{\star}$ where $\boldsymbol{b}$ is a vector in $\mathbb{N}^{d}$ and $\boldsymbol{P}$ is a finite subset of $\mathbb{N}^{d}$. The vector $\boldsymbol{b}$ is called the basis, and the vectors in $\boldsymbol{P}$ are called periods. A semilinear set is a finite union of linear sets. Recall that semilinear sets coincide with the sets definable in $\mathrm{FO}(\mathbb{N}, 0,1,+, \leq)$, also known as Presburger arithmetic [13].

Definition 3.1 ([13,14]). A finite subset $\boldsymbol{P}$ of $\mathbb{N}^{d}$ is stratified if every vector in $\boldsymbol{P}$ has at most two non-zero components, and it holds that

$$
\boldsymbol{p}(r) \neq 0 \wedge \boldsymbol{p}(t) \neq 0 \wedge \boldsymbol{q}(s) \neq 0 \wedge \boldsymbol{q}(u) \neq 0 \Rightarrow \neg(r<s<t<u)
$$

for every vectors $\boldsymbol{p}, \boldsymbol{q} \in \boldsymbol{P}$ and indices $r, s, t, u \in\{1, \ldots, d\}$.

Example 3.2. The following examples are from [14]. The set $\{(2,1,0),(0,3,3)\}$ is stratified, but the set $\{(1,1,1),(1,0,2)\}$ is not stratified, since $(1,1,1)$ has three non-zero components. The set $\{(3,0,0,2),(0,1,5,0),(4,7,0,0)\}$ is stratified, but the set $\{(2,0,3,0),(0,3,0,2)\}$ is not stratified.

We call a semilinear set stratifiable when it is a finite union of linear sets, each with a stratified set of periods. Formally, a semilinear set $S$ is stratifiable if there exits a finite family $\left\{\left(\boldsymbol{b}_{i}, \boldsymbol{P}_{i}\right)\right\}_{i \in I}$ of vectors $\boldsymbol{b}_{i}$ in $\mathbb{N}^{d}$ and finite stratified subsets $\boldsymbol{P}_{i}$ of $\mathbb{N}^{d}$ such that $S=\bigcup_{i \in I}\left(\boldsymbol{b}_{i}+\boldsymbol{P}_{i}^{\star}\right)$. The following lemma shows that stratifiable semilinear sets enjoy nice closure properties.

Lemma 3.3. The class of stratifiable semilinear sets is closed under union, under projection, under inverse projection, and under intersection with Cartesian products of intervals.

Proof. These closure properties are easily derived from the definition of stratifiable semilinear sets.

The stratifiability problem asks whether a given semilinear set $S$ is stratifiable. The decidability of the stratifiability problem was raised in [13,14], and has been open for nearly fifty years. Stratifiability is linked to the following characterization of bounded context-free languages.

We consider words over a finite alphabet $\Sigma$. Recall that a language $L \subseteq \Sigma^{*}$ is bounded if $L \subseteq \sigma_{1}^{*} \cdots \sigma_{d}^{*}$ for some words $\sigma_{1}, \ldots, \sigma_{d}$ in $\Sigma^{*}$. In his book [14], Ginsburg characterizes which bounded languages are context-free, in terms of semilinear sets. The reader is referred to [14] for further details.

Theorem 3.4 ([14, p. 162]). Consider a language $L \subseteq \sigma_{1}^{*} \cdots \sigma_{d}^{*}$, where each $\sigma_{i} \in \Sigma^{*}$. Then $L$ is context-free if, and only if, the set of all vectors $\left(n_{1}, \ldots, n_{d}\right)$ in $\mathbb{N}^{d}$ such that $\sigma_{1}^{n_{1}} \cdots \sigma_{d}^{n_{d}} \in L$ is a stratifiable semilinear set.

Example 3.5. Take $\Sigma=\{a, b, c\}$. The language $\left\{a^{n} b^{m} c^{n} \mid n, m \in \mathbb{N}\right\}$ is contextfree since the set $\left\{\left(n_{1}, n_{2}, n_{3}\right) \in \mathbb{N}^{3} \mid n_{1}=n_{3}\right\}$ is the linear set $\boldsymbol{P}^{\star}$ where $\boldsymbol{P}=\{(1,0,1),(0,1,0)\}$ is stratified. The language $\left\{a^{n} b^{n} c^{n} \mid n \in \mathbb{N}\right\}$ is known to be non-context-free. This means the semilinear set $\mathbb{N}(1,1,1)$ is not stratifiable. 


\section{Decidability of Stratifiability for Integral Polyhedra}

In this section, we show that stratifiability is decidable for a subclass of semilinear sets, namely the sets of integral solutions of finite systems of linear inequalities. Formally, an integral polyhedron is a set of the form $\left\{\boldsymbol{x} \in \mathbb{N}^{d} \mid \boldsymbol{A} \boldsymbol{x} \geq \boldsymbol{b}\right\}$ where $\boldsymbol{A} \in \mathbb{Z}^{n \times d}$ is a matrix and $\boldsymbol{b} \in \mathbb{Z}^{d}$ is a vector. Every linear system $\boldsymbol{A} \boldsymbol{x} \geq \boldsymbol{b}$ can be encoded into Presburger arithmetic, so integral polyhedra are semilinear sets. The stratifiability problem for integral polyhedra asks, given a matrix $\boldsymbol{A} \in \mathbb{Z}^{n \times d}$ and a vector $\boldsymbol{b} \in \mathbb{Z}^{d}$, both encoded in binary, whether the integral polyhedron $\left\{\boldsymbol{x} \in \mathbb{N}^{d} \mid \boldsymbol{A} \boldsymbol{x} \geq \boldsymbol{b}\right\}$ is stratifiable. The remainder of this section reduces this problem to a decision problem that only involves the matrix $\boldsymbol{A} \in \mathbb{Z}^{n \times d}$.

First, we show that stratifiability for integral polyhedra can be reduced to the particular case of homogeneous linear inequalities. Formally, an integral cone is a set of the form $\left\{\boldsymbol{x} \in \mathbb{N}^{d} \mid \boldsymbol{A} \boldsymbol{x} \geq \mathbf{0}\right\}$ where $\boldsymbol{A} \in \mathbb{Z}^{n \times d}$ is a matrix. The following lemma shows that every integral cone is a linear set, and provides a way to decompose integral polyhedra into integral cones.

Lemma 4.1 ([21, p. 237]). For every matrix $\boldsymbol{A} \in \mathbb{Z}^{n \times d}$ and every vector $\boldsymbol{b} \in \mathbb{Z}^{d}$, there exists two finite subsets $\boldsymbol{B}$ and $\boldsymbol{P}$ of $\mathbb{N}^{d}$ such that:

$$
\left\{\boldsymbol{x} \in \mathbb{N}^{d} \mid \boldsymbol{A} \boldsymbol{x} \geq \boldsymbol{b}\right\}=\boldsymbol{B}+\boldsymbol{P}^{\star} \quad \text { and } \quad \boldsymbol{P}^{\star}=\left\{\boldsymbol{x} \in \mathbb{N}^{d} \mid \boldsymbol{A} \boldsymbol{x} \geq \mathbf{0}\right\}
$$

We have considered so far integral solutions of finite systems of linear inequalities. To simplify our analysis, we now move from integers to rational numbers. A cone is a set of the form $\left\{\boldsymbol{x} \in \mathbb{Q}_{\geq 0}^{d} \mid \boldsymbol{A x} \geq \mathbf{0}\right\}$ where $\boldsymbol{A} \in \mathbb{Z}^{n \times d}$ is a matrix. Given a finite set $\boldsymbol{P}=\left\{\boldsymbol{p}_{1}, \ldots, \boldsymbol{p}_{k}\right\}$ of vectors in $\mathbb{N}^{d}$, we let $\boldsymbol{P}^{\triangleleft}$ denote the set of linear combinations of vectors in $\boldsymbol{P}$ with nonnegative rational coefficients, i.e., $\boldsymbol{P}^{\triangleleft}=\mathbb{Q}_{\geq 0} \boldsymbol{p}_{1}+\cdots+\mathbb{Q}_{\geq 0} \boldsymbol{p}_{k}$. Put differently, $\boldsymbol{P}^{\triangleleft}$ is defined as $\boldsymbol{P}^{\star}$ except that $\mathbb{Q} \geq 0 \boldsymbol{p}_{i}$ replaces $\mathbb{N} \boldsymbol{p}_{i}$. Observe that $\boldsymbol{P}^{\triangleleft}=\mathbb{Q}_{\geq 0} \boldsymbol{P}^{\star}$ for every finite subset $\boldsymbol{P}$ of $\mathbb{N}^{d}$. Let us recall the following well-known property.

Property 4.2 (Farkas-Minkowski-Weyl Theorem). A subset $\boldsymbol{X}$ of $\mathbb{Q}_{\geq 0}^{d}$ is a cone if, and only if, $\boldsymbol{X}=\boldsymbol{P}^{\triangleleft}$ for some finite subset $\boldsymbol{P}$ of $\mathbb{N}^{d}$.

In order to extract the asymptotic directions of an integral polyhedron, we associate to every finite subset $\boldsymbol{P} \subseteq \mathbb{N}^{d}$ a partial-order $\sqsubseteq_{\boldsymbol{P}}$ on $\mathbb{N}^{d}$ defined by $\boldsymbol{x} \sqsubseteq_{\boldsymbol{P}} \boldsymbol{y}$ if $\boldsymbol{y} \in \boldsymbol{x}+\boldsymbol{P}^{\star}$. Observe that when $\boldsymbol{P}=\left\{\boldsymbol{e}_{1}, \ldots, \boldsymbol{e}_{d}\right\}$, the partial-order $\sqsubseteq_{\boldsymbol{P}}$ coincides with the classical partial order $\leq$ on $\mathbb{N}^{d}$, which is known to be a well-partial-order on $\mathbb{N}^{d}=\mathbb{N}^{d} \cap \boldsymbol{P}^{\triangleleft}$, by Dickson's lemma. This observation can be generalized to any finite subset $\boldsymbol{P}$ of $\mathbb{N}^{d}$, as follows. We refer the reader to the proof of Lemma 1.2 from [15] for details.

Lemma 4.3 ([15]). The partial-order $\sqsubseteq_{\boldsymbol{P}}$ on $\mathbb{N}^{d}$ is a well-partial-order on $\mathbb{N}^{d} \cap \boldsymbol{P}^{\triangleleft}$, for every finite subset $\boldsymbol{P}$ of $\mathbb{N}^{d}$.

To show that the stratifiability problem for integral polyhedra is decidable, we decompose cones into "maximal stratifiable parts". The formal definition of 
these parts requires some additional notations. A binary relation $R$ on $\{1, \ldots, d\}$ is called nested if it satisfies the two following conditions:

$$
\begin{aligned}
(s, t) & \in R \Rightarrow s \leq t \\
(r, t) \in R \wedge(s, u) & \in R \Rightarrow \neg(r<s<t<u)
\end{aligned}
$$

An example of a nested relation is depicted in Figure 1a. Given a cone $\boldsymbol{X} \subseteq \mathbb{Q}_{\geq 0}^{d}$ and a nested binary relation $R$ on $\{1, \ldots, d\}$, we introduce the set $\boldsymbol{X}_{R}$ defined as follows:

$$
\boldsymbol{X}_{R}=\sum_{r \in R} \boldsymbol{X}_{r}
$$

where, for each pair $r=(s, t)$ of indices satisfying $1 \leq s \leq t \leq d$, the set $\boldsymbol{X}_{r}$ is given by:

$$
\boldsymbol{X}_{r}=\left\{\begin{array}{l|l}
\boldsymbol{x} \in \boldsymbol{X} & \bigwedge_{j \notin\{s, t\}} \boldsymbol{x}(j)=0
\end{array}\right\}
$$

Intuitively, the "maximal stratifiable parts" mentioned previously are the sets $\boldsymbol{X}_{R}$. The "stratification" property of $\boldsymbol{X}_{R}$ is expressed by the following lemma.

Lemma 4.4. For every cone $\boldsymbol{X} \subseteq \mathbb{Q}_{\geq 0}^{d}$ and every nested binary relation $R$ on $\{1, \ldots, d\}$, it holds that $\boldsymbol{X}_{R}=\boldsymbol{P}_{R}^{\triangleleft}$ for some finite stratified subset $\boldsymbol{P}_{R}$ of $\mathbb{N}^{d}$.

Proof. Consider a cone $\boldsymbol{X}=\left\{\boldsymbol{x} \in \mathbb{Q}_{>0}^{d} \mid \boldsymbol{A} \boldsymbol{x} \geq \mathbf{0}\right\}$ and a nested binary relation $R$ on $\{1, \ldots, d\}$. First, notice that $\boldsymbol{X}_{r}^{-}$is a cone for every $r=(s, t)$ in $R$. Indeed, every constraint $\boldsymbol{x}(j)=0$ may be expressed as the conjunction of $\boldsymbol{x}(j) \geq 0$ and $\boldsymbol{- x}(j) \geq 0$. By adding these inequalities to the matrix $\boldsymbol{A}$ for every $j \notin\{s, t\}$, we obtain a matrix witnessing that $\boldsymbol{X}_{r}$ is a cone. It follows from Property 4.2 that $\boldsymbol{X}_{r}=\boldsymbol{P}_{r}^{\triangleleft}$ for some finite subset $\boldsymbol{P}_{r} \subseteq \mathbb{N}^{d}$. Therefore, $\boldsymbol{X}_{R}=\boldsymbol{P}_{R}^{\triangleleft}$ where $\boldsymbol{P}_{R}$ is the finite subset of $\mathbb{N}^{d}$ defined by $\boldsymbol{P}_{R}=\bigcup_{r \in R} \boldsymbol{P}_{r}$. Since $R$ is nested, we derive that $\boldsymbol{P}_{R}$ is stratified, which concludes the proof of the lemma.

We are now ready to provide a decidable characterization of stratifiable integral polyhedra. Given a cone $\boldsymbol{X} \subseteq \mathbb{Q}_{>0}^{d}$, we say that $\boldsymbol{X}$ is nested if $\boldsymbol{X}=\bigcup_{R} \boldsymbol{X}_{R}$. It is understood that the union ranges over nested binary relations on $\{1, \ldots, d\}$. Observe that each $\boldsymbol{X}_{R}$ is contained in $\boldsymbol{X}$, since cones are closed under addition. So nestedness only requires that $\boldsymbol{X}$ is contained in $\bigcup_{R} \boldsymbol{X}_{R}$. The cone nestedness problem asks, given a matrix $\boldsymbol{A} \in \mathbb{Z}^{n \times d}$ encoded in binary, whether the cone $\left\{\boldsymbol{x} \in \mathbb{Q}_{\geq 0}^{d} \mid \boldsymbol{A} \boldsymbol{x} \geq \mathbf{0}\right\}$ is nested.

Theorem 4.5. An integral polyhedron $\left\{\boldsymbol{x} \in \mathbb{N}^{d} \mid \boldsymbol{A} \boldsymbol{x} \geq \boldsymbol{b}\right\}$ is stratifiable if, and only if, it is empty or the cone $\left\{\boldsymbol{x} \in \mathbb{Q}_{\geq 0}^{d} \mid \boldsymbol{A x} \geq \mathbf{0}\right\}$ is nested.

Proof. For brevity, we let $\boldsymbol{S}$ denote the integral polyhedron $\left\{\boldsymbol{x} \in \mathbb{N}^{d} \mid \boldsymbol{A} \boldsymbol{x} \geq \boldsymbol{b}\right\}$, and $\boldsymbol{X}$ denote its associated cone $\left\{\boldsymbol{x} \in \mathbb{Q}_{\geq 0}^{d} \mid \boldsymbol{A} \boldsymbol{x} \geq \mathbf{0}\right\}$.

We first prove the "if" direction of the theorem. If $\boldsymbol{S}$ is empty then it is trivially stratifiable. Let us assume that $\boldsymbol{X}$ is nested. According to Lemma 4.1, the integral 
polyhedron $\boldsymbol{S}$ can be decomposed into a sum $\boldsymbol{S}=\boldsymbol{B}+\boldsymbol{H}$ where $\boldsymbol{B} \subseteq \mathbb{N}^{d}$ is a finite set and $\boldsymbol{H}$ is the integral cone $\left\{\boldsymbol{x} \in \mathbb{N}^{d} \mid \boldsymbol{A x} \geq \mathbf{0}\right\}$. Observe that $\boldsymbol{X}=\bigcup_{R} \boldsymbol{X}_{R}$ since $\boldsymbol{X}$ is nested, and that $\boldsymbol{H} \subseteq \boldsymbol{X}$ by definition. We derive that $\boldsymbol{H}$ can be decomposed into $\boldsymbol{H}=\bigcup_{R} \boldsymbol{H}_{R}$ where $\boldsymbol{H}_{R}=\boldsymbol{H} \cap \boldsymbol{X}_{R}$. By Lemma 4.4, there exists a finite stratified set $\boldsymbol{P}_{R} \subseteq \mathbb{N}^{d}$ such that $\boldsymbol{X}_{R}=\boldsymbol{P}_{R}^{\triangleleft}$. Lemma 4.3 entails that $\sqsubseteq_{\boldsymbol{P}_{R}}$ is a well-partial-order on $\mathbb{N}^{d} \cap \boldsymbol{X}_{R}$. It follows that the set of minimal elements of $\boldsymbol{H}_{R}$ for this well-partial-order is a finite set. Moreover, writing $\boldsymbol{B}_{R}$ this finite set, we obtain that $\boldsymbol{H}_{R} \subseteq \boldsymbol{B}_{R}+\boldsymbol{P}_{R}^{\star}$. We have proved the inclusion $\boldsymbol{H} \subseteq \bigcup_{R}\left(\boldsymbol{B}_{R}+\boldsymbol{P}_{R}^{\star}\right)$. Since the converse inclusion is immediate, the set $\boldsymbol{H}$ is stratifiable. From $\boldsymbol{S}=\boldsymbol{B}+\boldsymbol{H}$, we deduce that $\boldsymbol{S}$ is stratifiable.

We now prove the "only if" direction of the theorem. Let us assume that $\boldsymbol{S}$ is non-empty and stratifiable. Since $\boldsymbol{S}$ is stratifiable, it can be decomposed into $\bigcup_{i \in I}\left(\boldsymbol{b}_{i}+\boldsymbol{P}_{i}^{\star}\right)$ where $I$ is finite, $\boldsymbol{b}_{i} \in \mathbb{N}^{d}$ and $\boldsymbol{P}_{i} \subseteq \mathbb{N}^{d}$ is a finite stratified set.

Let us first show that $\boldsymbol{P}_{i}^{\star} \subseteq \bigcup_{R} \boldsymbol{X}_{R}$. Define $R_{i}$ to be the set of pairs $(s, t)$, with $1 \leq s \leq t \leq d$, such that $\boldsymbol{p}(s) \neq 0 \wedge \boldsymbol{p}(t) \neq 0$ for some vector $\boldsymbol{p} \in \boldsymbol{P}_{i}$. It is readily seen that $R_{i}$ is a nested binary relation on $\{1, \ldots, d\}$, since $\boldsymbol{P}_{i}$ is stratified. Let $\boldsymbol{p} \in \boldsymbol{P}_{i}$. For each $n \in \mathbb{N}$, it holds that $\boldsymbol{b}_{i}+n \boldsymbol{p} \in \boldsymbol{S}$. Thus, $\boldsymbol{A} \boldsymbol{b}_{i}+n \boldsymbol{A} \boldsymbol{p} \geq \boldsymbol{b}$ for every $n \in \mathbb{N}$. We deduce that $\boldsymbol{A} \boldsymbol{p} \geq \mathbf{0}$, hence, $\boldsymbol{p} \in \boldsymbol{X}$. By definition of $R_{i}$, we get that $\boldsymbol{p} \in \boldsymbol{X}_{R_{i}}$. Since $\boldsymbol{X}_{R_{i}}$ is closed under addition, we obtain that $\boldsymbol{P}_{i}^{\star} \subseteq \boldsymbol{X}_{R_{i}}$.

To prove that $\boldsymbol{X} \subseteq \bigcup_{R} \boldsymbol{X}_{R}$, it is enough to show that $\mathbb{N}^{d} \cap \boldsymbol{X} \subseteq \bigcup_{R} \boldsymbol{X}_{R}$, since cones are closed under multiplication with nonnegative rational numbers. Let $\boldsymbol{x} \in \mathbb{N}^{d} \cap \boldsymbol{X}$. Since $\boldsymbol{S}$ is non-empty, there exists $\boldsymbol{s} \in \boldsymbol{S}$. For every $n \in \mathbb{N}$, we have $\boldsymbol{A}(\boldsymbol{s}+n \boldsymbol{x}) \geq \boldsymbol{b}$, hence, $\boldsymbol{s}+n \boldsymbol{x} \in \boldsymbol{S}$. By the pigeon-hole principle, there exists $i \in I$ and an infinite set $N \subseteq \mathbb{N}$ such that $\boldsymbol{s}+n \boldsymbol{x} \in \boldsymbol{b}_{i}+\boldsymbol{P}_{i}^{\star}$ for every $n \in N$. Lemma 4.3 entails that $\sqsubseteq_{\boldsymbol{P}_{i}}$ is a well-partial-order on $\mathbb{N}^{d} \cap \boldsymbol{P}_{i}^{\triangleleft}$. Since $\boldsymbol{x}_{n}=\boldsymbol{s}-\boldsymbol{b}_{i}+n \boldsymbol{x}$ is in $\boldsymbol{P}_{i}^{\star} \subseteq \mathbb{N}^{d} \cap \boldsymbol{P}_{i}^{\triangleleft}$ for every $n \in \mathbb{N}$, we deduce that there exists $n, m \in N$ such that $n<m$ and $\boldsymbol{x}_{n} \sqsubseteq \boldsymbol{P}_{i} \boldsymbol{x}_{m}$. It follows that $(m-n) \boldsymbol{x}=\boldsymbol{x}_{m}-\boldsymbol{x}_{n} \in \boldsymbol{P}_{i}^{\star}$. Since $\boldsymbol{P}_{i}^{\star} \subseteq \bigcup_{R} \boldsymbol{X}_{R}$, we obtain that $\boldsymbol{x} \in \bigcup_{R} \boldsymbol{X}_{R}$. We have shown that $\mathbb{N}^{d} \cap \boldsymbol{X} \subseteq \bigcup_{R} \boldsymbol{X}_{R}$, and we conclude that $\boldsymbol{X}$ is nested.

Given a cone $\boldsymbol{X}=\left\{\boldsymbol{x} \in \mathbb{Q}_{\geq 0}^{d} \mid \boldsymbol{A x} \geq \mathbf{0}\right\}$, we may compute from the matrix $\boldsymbol{A}$ a formula in the theory FO $(\mathbb{Q}, 0,1,+, \leq)$ expressing the equality $\boldsymbol{X}=\bigcup_{R} \boldsymbol{X}_{R}$. Since this theory is decidable by Fourier-Motzkin quantifier elimination, we obtain that the cone nestedness problem is decidable. It follows from Theorem 4.5 that the stratifiability problem for integral polyhedra is decidable. We show, in the next section, that this problem is coNP-complete.

\section{A Criterion for Cone Nestedness Decidable in coNP}

We provide, in this section, a criterion for checking whether a given cone is nested. This criterion leads to a coNP decision procedure for the cone nestedness problem, and similarly for the stratifiability problem for integral polyhedra. We also show that the latter upper bound is tight.

To every cone $\boldsymbol{X} \subseteq \mathbb{Q}_{\geq 0}^{d}$, we associate the set $\overparen{\boldsymbol{X}}=\bigcup_{R} \boldsymbol{X}_{R}$. Recall that $\boldsymbol{X}$ is non-nested precisely when $\boldsymbol{X} \nsubseteq \overparen{\boldsymbol{X}}$. We show that non-nestedness of a cone $\boldsymbol{X}$ 


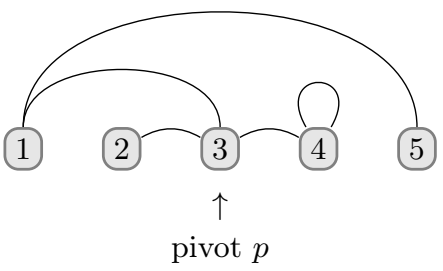

(a) Nested relation

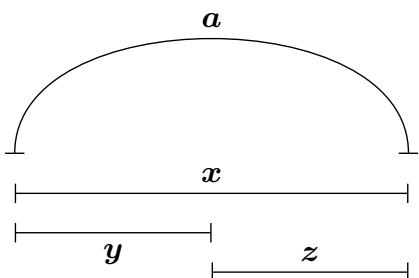

(b) Reducible vector

Fig. 1: Decomposition of nested relations and of reducible vectors

can always be witnessed by a vector in $\boldsymbol{X} \backslash \overparen{\boldsymbol{X}}$ of a special form. We will need the following easy facts. An illustration of the first lemma is given in Figure 1a.

Lemma 5.1. Assume that $d \geq 3$. A binary relation $R$ on $\{1, \ldots, d\}$ is nested if, and only if, there exist a pivot $p$ with $1<p<d$ and two nested binary relations $U$ and $V$ on $\{1, \ldots, p\}$ and $\{p, \ldots, d\}$, respectively, such that $R \subseteq\{(1, d)\} \cup U \cup V$.

Lemma 5.2. For every $\boldsymbol{x} \in \overparen{\boldsymbol{X}}$, there exists a nested binary relation $R$ on ${ }^{3}$ $\operatorname{supp}(\boldsymbol{x})$ such that $\boldsymbol{x} \in \boldsymbol{X}_{R}$.

Proof (sketch). If $\boldsymbol{x} \in \overparen{\boldsymbol{X}}$ then $\boldsymbol{x}=\sum_{r \in R} \boldsymbol{x}_{r}$ for some nested binary relation $R$ on $\{1, \ldots, d\}$ and some vectors $\boldsymbol{x}_{r} \in \boldsymbol{X}_{r}$. We can assume, w.l.o.g., that $\boldsymbol{x}_{r}(s) \neq 0$ and $\boldsymbol{x}_{r}(t) \neq 0$ for each pair $r=(s, t)$. Indeed, if $\boldsymbol{x}_{r}=\mathbf{0}$ then $r$ can be removed from $R$, and, otherwise, if $\boldsymbol{x}_{r}(s)=0$ or $\boldsymbol{x}_{r}(t)=0$ then $r$ can be replaced by $(t, t)$ or $(s, s)$, respectively. It follows that $R$ is a nested binary relation on $\operatorname{supp}(\boldsymbol{x})$.

Consider a cone $\boldsymbol{X} \subseteq \mathbb{Q}_{\geq 0}^{d}$. Given a pair $r=(s, t)$ of indices with $1 \leq s \leq t \leq d$, we call $r$-decomposition ${ }^{4}$ any triple $(\boldsymbol{a}, \boldsymbol{y}, \boldsymbol{z})$ of vectors in $\boldsymbol{X}_{r} \times \boldsymbol{X} \times \boldsymbol{X}$ such that $\operatorname{supp}(\boldsymbol{y}) \subseteq\{s, \ldots, p\}$ and $\operatorname{supp}(\boldsymbol{z}) \subseteq\{p, \ldots, t\}$ for some pivot $p$ satisfying $s<p<t$. Since cones are closed under addition, the vector $\boldsymbol{a}+\boldsymbol{y}+\boldsymbol{z}$ is in $\boldsymbol{X}$ for every decomposition $(\boldsymbol{a}, \boldsymbol{y}, \boldsymbol{z})$. The following lemma shows that membership in $\overparen{\boldsymbol{X}}$ is also preserved by decomposition.

Lemma 5.3. For every $r$-decomposition $(\boldsymbol{a}, \boldsymbol{y}, \boldsymbol{z})$, it holds that $(\boldsymbol{a}+\boldsymbol{y}+\boldsymbol{z}) \in \overparen{\boldsymbol{X}}$ if $\boldsymbol{y} \in \overparen{\boldsymbol{X}}$ and $\boldsymbol{z} \in \overparen{\boldsymbol{X}}$.

Proof. Assume that $\boldsymbol{y} \in \overparen{\boldsymbol{X}}$ and $\boldsymbol{z} \in \overparen{\boldsymbol{X}}$. By Lemma 5.2, there exists two nested binary relations $U$ on $\operatorname{supp}(\boldsymbol{y})$ and $V$ on $\operatorname{supp}(\boldsymbol{z})$ such that $\boldsymbol{y} \in \boldsymbol{X}_{U}$ and $\boldsymbol{z} \in \boldsymbol{X}_{V}$. Since $(\boldsymbol{a}, \boldsymbol{y}, \boldsymbol{z})$ is an $r$-decomposition, there exists a pivot $p$ with $s<p<t$, where $r=(s, t)$, such that $\operatorname{supp}(\boldsymbol{y}) \subseteq\{s, \ldots, p\}$ and $\operatorname{supp}(\boldsymbol{z}) \subseteq\{p, \ldots, t\}$. It follows that the field ${ }^{5}$ of $U$ and the field of $V$ are contained in $\{s, \ldots, p\}$ and

\footnotetext{
${ }^{3}$ Recall that $\operatorname{supp}(\boldsymbol{v})$ denotes the support of $\boldsymbol{v}$, i.e, the set of indices $i$ with $\boldsymbol{v}(i) \neq 0$.

${ }^{4}$ This notion is defined relative to a cone $\boldsymbol{X}$, which is left implicit to reduce clutter.

${ }^{5}$ The field of a binary relation is the union of its domain and range.
} 
$\{p, \ldots, t\}$, respectively. We derive from Lemma 5.1 that the binary relation $R=\{(s, t)\} \cup U \cup V$ is nested. Observe that $\boldsymbol{a}, \boldsymbol{y}$ and $\boldsymbol{z}$ are all in $\boldsymbol{X}_{R}$. Since $\boldsymbol{X}_{R}$ is closed under addition, we obtain that $(\boldsymbol{a}+\boldsymbol{y}+\boldsymbol{z}) \in \boldsymbol{X}_{R}$, which concludes the proof of the lemma.

We call a vector $\boldsymbol{x} \in \boldsymbol{X}$ reducible $e^{4}$ when $\operatorname{supp}(\boldsymbol{x})$ has cardinality at most two, or there exists a pair $(s, t)$ of indices in $\operatorname{supp}(\boldsymbol{x})$, with $1 \leq s \leq t \leq d$, and an $(s, t)$-decomposition $(\boldsymbol{a}, \boldsymbol{y}, \boldsymbol{z})$ such that $\boldsymbol{x}=\boldsymbol{a}+\boldsymbol{y}+\boldsymbol{z}$. The latter condition is depicted in Figure 1b. Note that this condition entails that $\operatorname{supp}(\boldsymbol{x}) \subseteq\{s, \ldots, t\}$. A vector $\boldsymbol{x} \in \boldsymbol{X}$ is called irreducible ${ }^{4}$ when it is not reducible. The following theorem characterizes which cones are nested, in terms of irreducible vectors. Before that, we illustrate these notions on a few examples.

Example 5.4. Let $\boldsymbol{X}$ and $\boldsymbol{Y}$ be the cones given by $\boldsymbol{X}=\left\{\boldsymbol{x} \in \mathbb{Q}_{>0}^{3} \mid \boldsymbol{x}(1)=\boldsymbol{x}(3)\right\}$ and $\boldsymbol{Y}=\left\{\boldsymbol{x} \in \mathbb{Q}_{\geq 0}^{3} \mid \boldsymbol{x}(1)=\boldsymbol{x}(2)=\boldsymbol{x}(3)\right\}$. The vector $(1,0,1)$ is reducible for both cones, since it contains only two non-zero components. The vector $(1,1,1)$ is reducible for $\boldsymbol{X}$. This is witnessed by the (1,3)-decomposition $(\boldsymbol{a}, \boldsymbol{y}, \boldsymbol{y})$ where $\boldsymbol{a}=(1,0,1)$ and $\boldsymbol{y}=(0,0.5,0)$. The same vector $(1,1,1)$ is irreducible for $\boldsymbol{Y}$.

Theorem 5.5. A cone is nested if, and only if, it contains no irreducible vector.

Proof. Consider a cone $\boldsymbol{X} \subseteq \mathbb{Q}_{>0}^{d}$. We first prove the "only if" direction of the theorem. Assume that $\boldsymbol{X}$ is nested, and let $\boldsymbol{x} \in \boldsymbol{X}$. If $\operatorname{supp}(\boldsymbol{x})$ has cardinality at most two, then $\boldsymbol{x}$ is trivially reducible. Suppose, on the contrary, that $\boldsymbol{x}$ contains at least three non-zero components. Since $\boldsymbol{X} \subseteq \widehat{\boldsymbol{X}}$, we obtain from Lemma 5.2 that there exists a nested binary relation $R$ on $\operatorname{supp}(\boldsymbol{x})$ such that $\boldsymbol{x} \in \boldsymbol{X}_{R}$. Moreover, $R$ is not empty since $\boldsymbol{x} \neq \mathbf{0}$. Let $F$ denote the field ${ }^{5}$ of $R$, and define $s=\min F$ and $t=\max F$. Observe that both $s$ and $t$ are in $\operatorname{supp}(\boldsymbol{x})$. Notice also that $t \geq s+2$ since $\boldsymbol{x}$ contains at least three non-zero components. We derive from Lemma 5.1 that there exist a pivot $p$ with $s<p<t$, a nested binary relation $U$ on $\{s, \ldots, p\}$ and a nested binary relation $V$ on $\{p, \ldots, t\}$, such that $R \subseteq\{(s, t)\} \cup U \cup V$. We derive that $\boldsymbol{x} \in\left(\boldsymbol{X}_{(s, t)}+\boldsymbol{X}_{U}+\boldsymbol{X}_{V}\right)$, which entails that $\boldsymbol{x}$ is reducible.

Let us now prove the "if" direction of the theorem. Assume that $\boldsymbol{X}$ is not nested. This means that $\boldsymbol{X} \nsubseteq \widehat{\boldsymbol{X}}$. Among the vectors $\boldsymbol{x}$ in $\boldsymbol{X} \backslash \widehat{\boldsymbol{X}}$, pick one such that $\operatorname{supp}(\boldsymbol{x})$ is minimal for inclusion. Let us show that $\boldsymbol{x}$ is irreducible. By contradiction, suppose that $\boldsymbol{x}$ is reducible. Observe that $\operatorname{supp}(\boldsymbol{x})$ has cardinality at least three since $\boldsymbol{x} \in(\boldsymbol{X} \backslash \widehat{\boldsymbol{X}})$. Therefore, there exists a pair $(s, t)$ of indices in $\operatorname{supp}(\boldsymbol{x})$, with $1 \leq s \leq t \leq d$, and an $(s, t)$-decomposition $(\boldsymbol{a}, \boldsymbol{y}, \boldsymbol{z})$ such that $\boldsymbol{x}=\boldsymbol{a}+\boldsymbol{y}+\boldsymbol{z}$. It is readily seen that $\operatorname{supp}(\boldsymbol{y})$ and $\operatorname{supp}(\boldsymbol{z})$ are both strictly contained in $\operatorname{supp}(\boldsymbol{x})$. By minimality of $\boldsymbol{x}$, we get that $\boldsymbol{y}$ and $\boldsymbol{z}$ are in $\widehat{\boldsymbol{X}}$. We derive from Lemma 5.3 that $\boldsymbol{x} \in \widehat{\boldsymbol{X}}$, which contradicts the assumption that $\boldsymbol{x}$ is in $\boldsymbol{X} \backslash \widehat{\boldsymbol{X}}$.

The previous theorem allows us to reduce the cone nestedness problem to the question whether a given cone contains only reducible vectors. In the remainder 
of this section, we explain how to solve the latter problem in coNP. Consider a matrix $\boldsymbol{A} \in \mathbb{Z}^{n \times d}$ encoded in binary, and let $\boldsymbol{X}=\left\{\boldsymbol{x} \in \mathbb{Q}_{\geq 0}^{d} \mid \boldsymbol{A x} \geq \mathbf{0}\right\}$. We build, in time polynomial in the size of $\boldsymbol{A}$, a quantifier-free formula ${ }^{6} \rho(\boldsymbol{x})$ in FO $(\mathbb{Q}, 0,1,+, \leq)$ that is valid if, and only if, $\boldsymbol{X}$ contains only reducible vectors. This will entail a coNP upper bound for the cone nestedness problem, since satisfiability of quantifier-free formulas in $\mathrm{FO}(\mathbb{Q}, 0,1,+, \leq)$ is solvable in NP (see, e.g., [21, p. 120]). First, we build a formula $\varphi(\boldsymbol{x})$, containing quantifiers, whose models are precisely the vectors in $\boldsymbol{X}$ that are reducible. Let $\boldsymbol{B}$ be the the matrix in $\mathbb{Z}^{(n+d) \times d}$ obtained from $\boldsymbol{A}$ by appending the identity matrix to the bottom of $\boldsymbol{A}$. Note that $\boldsymbol{X}=\left\{\boldsymbol{x} \in \mathbb{Q}^{d} \mid \boldsymbol{B} \boldsymbol{x} \geq \mathbf{0}\right\}$. The formula $\varphi(\boldsymbol{x})$ is:

$\boldsymbol{B} \boldsymbol{x} \geq \mathbf{0} \wedge \bigvee_{1 \leq s \leq t \leq d}\left[\left(\bigwedge_{i \notin\{s, t\}} \boldsymbol{x}(i)=0\right) \vee\left(\boldsymbol{x}(s)>0 \wedge \boldsymbol{x}(t)>0 \wedge \psi_{\boldsymbol{B}, s, t}(\boldsymbol{x})\right)\right]$

where, for each pair $(s, t)$ of indices with $1 \leq s \leq t \leq d$, the formula $\psi_{\boldsymbol{B}, s, t}(\boldsymbol{x})$, given below, expresses that there exists an $(s, t)$-decomposition $(\boldsymbol{a}, \boldsymbol{y}, \boldsymbol{z})$ such that $\boldsymbol{x}=\boldsymbol{a}+\boldsymbol{y}+\boldsymbol{z}$. The formula $\psi_{\boldsymbol{B}, s, t}(\boldsymbol{x})$ is:

$\left(\bigwedge_{i<s \vee i>t} \boldsymbol{x}(i)=0\right) \wedge \bigvee_{p=s+1}^{t-1} \exists \mu \exists \nu \exists \pi\left[\begin{array}{l}\boldsymbol{B}\left(\mu \boldsymbol{e}_{s}+\nu \boldsymbol{e}_{t}\right) \\ \boldsymbol{B}\left(\sum_{s \leq i<p} \boldsymbol{x}(i) \boldsymbol{e}_{i}-\mu \boldsymbol{e}_{s}+\pi \boldsymbol{e}_{p}\right) \geq \mathbf{0} \\ \boldsymbol{B}\left(\sum_{p \leq i \leq t} \boldsymbol{x}(i) \boldsymbol{e}_{i}-\pi \boldsymbol{e}_{p}-\nu \boldsymbol{e}_{t}\right) \geq \mathbf{0}\end{array}\right]$

Here, it is understood that the sub-formula in brackets stands for the conjunction of the three systems of linear inequalities. It is routinely checked that, for every vector $\boldsymbol{x} \in \mathbb{Q}^{d}$, the formula $\varphi(\boldsymbol{x})$ holds if, and only if, $\boldsymbol{x}$ is a reducible vector of $\boldsymbol{X}$. Notice that each disjunct $\exists \mu \exists \nu \exists \pi[\cdots]$ contains a constant number of quantifiers, namely three. So, with Fourier-Motzkin quantifier elimination, we can transform, in polynomial time, each formula $\psi_{\boldsymbol{B}, s, t}(\boldsymbol{x})$ into an equivalent quantifier-free formula $\psi_{\boldsymbol{B}, s, t}^{\prime}(\boldsymbol{x})$. Let $\varphi^{\prime}(\boldsymbol{x})$ denote the formula obtained from the definition of $\varphi(\boldsymbol{x})$ by replacing each $\psi_{\boldsymbol{B}, s, t}(\boldsymbol{x})$ by $\psi_{\boldsymbol{B}, s, t}^{\prime}(\boldsymbol{x})$. The desired formula $\rho(\boldsymbol{x})$ is $(\boldsymbol{B} \boldsymbol{x} \geq \mathbf{0}) \Rightarrow \varphi^{\prime}(\boldsymbol{x})$. We have shown the following theorem.

Theorem 5.6. The cone nestedness problem is solvable in coNP.

We now have all the necessary ingredients to prove the following corollary, which is the main technical result of the paper.

Corollary 5.7. The stratifiability problem for integral polyhedra is coNP-complete.

Proof. We start by recalling that the emptiness problem for integral polyhedra is coNP-complete (see, e.g., [21, p. 245]). This problem asks, given a matrix

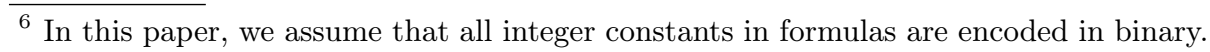


$\boldsymbol{A} \in \mathbb{Z}^{n \times d}$ and a vector $\boldsymbol{b} \in \mathbb{Z}^{d}$, both encoded in binary, whether the integral polyhedron $\left\{\boldsymbol{x} \in \mathbb{N}^{d} \mid \boldsymbol{A} \boldsymbol{x} \geq \boldsymbol{b}\right\}$ is empty.

Let us now prove the corollary. The upper bound follows from Theorem 4.5, Theorem 5.6, and closure under union of coNP. The lower bound is obtained by reduction from the emptiness problem for integral polyhedra. First of all, we observe that by increasing $d$ by 3 and by slightly modifying the pair $(\boldsymbol{A}, \boldsymbol{b})$, the emptiness problem for integral polyhedra can be reduced, in linear time, to the particular case of integral polyhedra $\boldsymbol{X}$ satisfying the following condition:

$$
\boldsymbol{X}=\emptyset \quad \text { or } \quad\{(\boldsymbol{x}(1), \boldsymbol{x}(2), \boldsymbol{x}(3)) \mid \boldsymbol{x} \in \boldsymbol{X}\}=\mathbb{N}(1,1,1)
$$

Recall that the linear set $\mathbb{N}(1,1,1)$ is not stratifiable (see Example 3.5). It follows from Lemma 3.3 that every integral polyhedron satisfying (3) is empty if, and only if, it is stratifiable. We have thus reduced, in linear time, the emptiness problem for integral polyhedra to the stratifiability problem for them.

\section{Application to Flat Counter Systems}

In this section, we investigate the context-freeness problem for flat counter systems. This problem asks whether the trace language of a given counter system intersected with a given bounded regular language is context-free. In our setting, counter systems are a generalization of Petri nets where transitions are guarded by integral polyhedra. Such guards can express zero tests, so counter systems subsume Minsky machines and, therefore, are Turing-powerful. We show that the context-freeness problem for flat counter systems is coNP-complete, and remains so for flat Petri nets.

We exploit the restriction to bounded languages required by flatness to reduce the context-freeness problem for flat counter systems to the stratifiability problem for integral polyhedra. This reduction is performed in two steps. It is well-known that bounded regular languages are finite unions of languages of the form $w_{1} \sigma_{1}^{+} \cdots w_{d} \sigma_{d}^{+}[12]$. As a first step, we consider a subproblem of the context-freeness problem for flat counter systems, where the given bounded regular language is of the form $w_{1} \sigma_{1}^{+} \cdots w_{d} \sigma_{d}^{+}$. We provide a reduction of this subproblem to the stratifiability problem for integral polyhedra. The contextfreeness problem for flat counter systems is then reduced to this subproblem by providing polynomial bounds on the size of the languages $w_{1} \sigma_{1}^{+} \cdots w_{d} \sigma_{d}^{+}$.

A counter system is a formal model manipulating a finite set of counters ranging over the natural numbers. Given a number of counters $c \in \mathbb{N}$, a configuration is a vector $\boldsymbol{x} \in \mathbb{N}^{c}$, and a transition is a triple $\theta=(\boldsymbol{A}, \boldsymbol{b}, \boldsymbol{v})$ where $\boldsymbol{A} \in \mathbb{Z}^{m \times c}$ is a matrix, and $\boldsymbol{b} \in \mathbb{Z}^{m}$ and $\boldsymbol{v} \in \mathbb{Z}^{c}$ are two vectors. Informally, a transition $(\boldsymbol{A}, \boldsymbol{b}, \boldsymbol{v})$ represents the guarded translation " $\boldsymbol{A} \boldsymbol{x} \geq \boldsymbol{b} ; \boldsymbol{x}:=\boldsymbol{x}+\boldsymbol{v} ; \boldsymbol{x} \geq \mathbf{0}$ ".

Formally, a counter system is a triple $\mathcal{S}=\left\langle c, \Theta, \boldsymbol{x}_{\text {init }}\right\rangle$ where $c \in \mathbb{N}$ is a number of counters, $\Theta \subseteq \mathbb{Z}^{m \times c} \times \mathbb{Z}^{m} \times \mathbb{Z}^{c}$ is a finite set of transitions, and $\boldsymbol{x}_{\text {init }} \in \mathbb{N}^{c}$ is an initial configuration. The operational semantics of $\mathcal{S}$ is given by the labeled 
transition relation $\rightarrow \subseteq \mathbb{N}^{c} \times \Theta \times \mathbb{N}^{c}$, defined by $\boldsymbol{x} \stackrel{(\boldsymbol{A}, \boldsymbol{b}, \boldsymbol{v})}{\longrightarrow} \boldsymbol{y}$ if $\boldsymbol{A} \boldsymbol{x} \geq \boldsymbol{b}$ and $\boldsymbol{y}=\boldsymbol{x}+\boldsymbol{v}$. A run is a finite, alternating sequence $\left(\boldsymbol{x}_{0}, \theta_{1}, \boldsymbol{x}_{1}, \ldots, \theta_{k}, \boldsymbol{x}_{k}\right)$ of configurations and transitions, satisfying $\boldsymbol{x}_{i-1} \stackrel{\theta_{i}}{\longrightarrow} \boldsymbol{x}_{i}$ for all $i$. The word $\theta_{1} \cdots \theta_{k}$ is called the label of the run. We introduce, for every word $\sigma \in \Theta^{*}$, the binary relation $\stackrel{\sigma}{\rightarrow}$ between configurations, defined by $\boldsymbol{x} \stackrel{\sigma}{\rightarrow} \boldsymbol{y}$ if there exists a run from $\boldsymbol{x}$ to $\boldsymbol{y}$ labeled by $\sigma$. A trace from a configuration $\boldsymbol{x}$ is the label of some run that starts with $\boldsymbol{x}$. We let $\mathcal{T}(\mathcal{S}, \boldsymbol{x})$ denote the set of all traces from $\boldsymbol{x}$. The set $\mathcal{T}\left(\mathcal{S}, \boldsymbol{x}_{\text {init }}\right)$ of all traces from the initial configuration, shortly written $\mathcal{T}(\mathcal{S})$, is called the trace language of $\mathcal{S}$. The context-freeness problem for counter systems asks whether the trace language of a given counter system is context-free. This problem is easily shown to be undecidable, by reduction from the reachability problem for (deterministic) Minsky machines.

For the subclass of Petri nets, the context-freeness problem was shown to be decidable by Schwer in [22]. In our settings, a Petri net is a counter system $\left\langle c, \Theta, \boldsymbol{x}_{\text {init }}\right\rangle$ where $\Theta$ is a set of transitions of the form $(\boldsymbol{A}, \boldsymbol{b}, \boldsymbol{v})$ such that $\boldsymbol{A}$ is the identity matrix. Informally, Petri net transitions are guarded translations " $\boldsymbol{x} \geq \boldsymbol{b} ; \boldsymbol{x}:=\boldsymbol{x}+\boldsymbol{v} ; \boldsymbol{x} \geq \mathbf{0}$ ". In a recent paper [18], we revisited the contextfreeness problem for Petri nets, and gave a simpler proof of decidability based on bounded regular languages. We showed that the trace language of a Petri net is context-free if, and only if, it has a context-free intersection with every bounded regular language. Based on this characterization, the context-freeness problem for Petri nets was then shown to be ExPSPACE-complete [19]. However, the complexity of the context-freeness problem for flat Petri nets was left open.

This motivates our study of the context-freeness problem for flat counter systems. Formally, we define this problem as follows:

Input: a counter system $\mathcal{S}=\left\langle c, \Theta, \boldsymbol{x}_{\text {init }}\right\rangle$, and a finite-state automaton ${ }^{7} \mathcal{A}$ recognizing a bounded regular language $L(\mathcal{A}) \subseteq \Theta^{*}$,

Output: whether the language $\mathcal{T}(\mathcal{S}) \cap L(\mathcal{A})$ is context-free.

The size of the input is the obvious one, where integers are encoded in binary. In the sequel, this problem is reduced to a subproblem, called the context-freeness problem for flat-linear counter systems, which is defined as follows:

Input: a counter system $\mathcal{S}=\left\langle c, \Theta, \boldsymbol{x}_{\text {init }}\right\rangle$, and a finite sequence $w_{1}, \sigma_{1}, \ldots, w_{d}, \sigma_{d}$ of words in $\Theta^{*}$,

Output: whether the language $\mathcal{T}(\mathcal{S}) \cap w_{1} \sigma_{1}^{+} \cdots w_{d} \sigma_{d}^{+}$is context-free.

The size of the input is, again, the obvious one, with integers encoded in binary. The decidability of this last problem requires the following variant of Ginsburg's characterization of bounded context-free languages (cf. Theorem 3.4).

\footnotetext{
${ }^{7}$ Recall that a finite-state automaton is a quintuple $\mathcal{A}=\langle Q, I, F, \Sigma, \rightarrow\rangle$ where $Q$ is a finite set of states, $I \subseteq Q$ and $F \subseteq Q$ are finite sets of initial and final states, $\Sigma$ is a finite alphabet, and $\rightarrow \subseteq Q \times \Sigma \times Q$ is a finite set of transitions. We let $L(\mathcal{A})$ denote the language recognized by $\mathcal{A}$.
} 
Lemma 6.1. Consider a language $L \subseteq w_{1} \sigma_{1}^{+} \cdots w_{d} \sigma_{d}^{+}$, where each $w_{i} \in \Sigma^{*}$ and each $\sigma_{i} \in \Sigma^{*}$. Then $L$ is context-free if, and only if, the set of all vectors $\left(n_{1}, \ldots, n_{d}\right)$ in $\mathbb{N}_{1}^{d}$ such that $w_{1} \sigma_{1}^{n_{1}} \cdots w_{d} \sigma_{d}^{n_{d}} \in L$ is a stratifiable semilinear set.

The context-freeness problem for flat-linear counter systems is shown to be decidable in coNP by a polynomial-time reduction to the stratifiability problem for integral polyhedra, which is solvable in coNP by Corollary 5.7. Let us consider an input of this problem, namely a counter system $\mathcal{S}=\left\langle c, \Theta, \boldsymbol{x}_{\text {init }}\right\rangle$ and a finite sequence $w_{1}, \sigma_{1}, \ldots, w_{d}, \sigma_{d}$ of words in $\Theta^{*}$. By Lemma 6.1, the language $L=\mathcal{T}(\mathcal{S}) \cap w_{1} \sigma_{1}^{+} \cdots w_{d} \sigma_{d}^{+}$is context-free if, and only if, the following set is stratifiable:

$$
\boldsymbol{N}=\left\{\left(n_{1}, \ldots, n_{d}\right) \in \mathbb{N}_{1}^{d} \mid w_{1} \sigma_{1}^{n_{d}} \cdots w_{d} \sigma_{d}^{n_{d}} \in \mathcal{T}(\mathcal{S})\right\}
$$

This set is shown to be an integral polyhedron in Corollary 6.3. This result follows from the following "acceleration" lemma.

Lemma 6.2. There exists a polynomial-time algorithm that, given a counter system $\mathcal{S}=\left\langle c, \Theta, \boldsymbol{x}_{\text {init }}\right\rangle$ and a word $\sigma \in \Theta^{*}$, computes a matrix $\boldsymbol{A} \in \mathbb{Z}^{n \times c}$ and three vectors $\boldsymbol{a}, \boldsymbol{b} \in \mathbb{Z}^{n}$ and $\boldsymbol{v} \in \mathbb{Z}^{c}$ such that:

$$
\boldsymbol{x} \stackrel{\sigma^{n}}{\longrightarrow} \boldsymbol{y} \Longleftrightarrow \boldsymbol{A} \boldsymbol{x}+n \boldsymbol{a} \geq \boldsymbol{b} \wedge \boldsymbol{y}=\boldsymbol{x}+n \boldsymbol{v}
$$

for every $\boldsymbol{x}, \boldsymbol{y} \in \mathbb{N}^{c}$ and $n \in \mathbb{N}_{1}$.

Proof (sketch). By encoding the effect of a word $\sigma \in \Theta^{*}$ into a single transition, we deduce the lemma thanks to [2]. The crucial observation is the convexity of the guard of this transition.

Corollary 6.3. There exists a polynomial-time algorithm that, given a counter system $\mathcal{S}=\left\langle c, \Theta, \boldsymbol{x}_{\text {init }}\right\rangle$ and a sequence $w_{1}, \sigma_{1}, \ldots, w_{d}, \sigma_{d}$ of words in $\Theta^{*}$, computes a matrix $\boldsymbol{A} \in \mathbb{Z}^{n \times d}$ and a vector $\boldsymbol{b} \in \mathbb{Z}^{n}$ such that:

$$
w_{1} \sigma_{1}^{n_{1}} \cdots w_{d} \sigma_{d}^{n_{d}} \in \mathcal{T}(\mathcal{S}) \Longleftrightarrow \boldsymbol{A}\left(n_{1}, \ldots, n_{d}\right) \geq \boldsymbol{b}
$$

for every $n_{1}, \ldots, n_{d} \in \mathbb{N}_{1}$.

Proof. We derive in polynomial time from Lemma 6.2, a tuple $\left(\boldsymbol{A}_{i}, \boldsymbol{a}_{i}, \boldsymbol{b}_{i}, \boldsymbol{v}_{i}\right)$ such that for every $n \in \mathbb{N}_{1}$ and any $\boldsymbol{x}, \boldsymbol{y} \in \mathbb{N}^{c}$ we have $\boldsymbol{x} \stackrel{\sigma_{i}^{n}}{\longrightarrow} \boldsymbol{y}$ if, and only if, $\boldsymbol{A}_{i} \boldsymbol{x}+n \boldsymbol{a}_{i} \geq \boldsymbol{b}_{i}$ and $\boldsymbol{y}=\boldsymbol{x}+n_{i} \boldsymbol{v}_{i}$. In polynomial time, we compute transitions $\theta_{i}=\left(\boldsymbol{B}_{i}, \boldsymbol{c}_{i}, \boldsymbol{u}_{i}\right)$ such that the binary relation $\stackrel{w_{i}}{\longrightarrow}$ is equal to $\stackrel{\theta_{i}}{\longrightarrow}$. The word $w_{1} \sigma_{1}^{n_{1}} \cdots w_{d} \sigma_{d}^{n_{d}}$ is a trace from $\boldsymbol{x}_{\text {init }}$ with $n_{1}, \ldots, n_{d} \in \mathbb{N}_{1}$ if, and only if, the following linear system is satisfiable where $\boldsymbol{y}_{i}=\boldsymbol{x}_{\mathrm{init}}+\sum_{1 \leq j<i}\left(\boldsymbol{u}_{j}+n_{j} \boldsymbol{v}_{j}\right)$ and $\boldsymbol{x}_{i}=\boldsymbol{y}_{i}+\boldsymbol{u}_{i}$ :

$$
\bigwedge_{i=1}^{d} \boldsymbol{B}_{i} \boldsymbol{y}_{i} \geq \boldsymbol{c}_{i} \wedge \boldsymbol{A}_{i} \boldsymbol{x}_{i}+n_{i} \boldsymbol{a}_{i} \geq \boldsymbol{b}_{i}
$$

Now, just observe that such a linear system can be written as a linear system of the form $\boldsymbol{A}\left(n_{1}, \ldots, n_{d}\right) \geq \boldsymbol{b}$. 
We deduce the following theorem.

Theorem 6.4. The context-freeness problem for flat-linear counter systems is coNP-complete.

Proof. Since the language $L$ is context-free if, and only if, the integral polyhedron $N$ is stratifiable, it follows from Corollary 5.7 that the context-freeness problem for flat-linear counter systems is in coNP. The problem is shown to be coNP-hard by a direct reduction from the stratifiability problem for integral polyhedra, which is coNP-hard by Corollary 5.7 .

The context-freeness problem for flat counter systems can be reduced to the flat-linear case thanks to the following lemma, which provides polynomial bounds on the decomposition of bounded regular languages into languages of the form $w_{1} \sigma_{1}^{+} \cdots w_{d} \sigma_{d}^{+}$.

Lemma 6.5. Let $\mathcal{A}$ be a finite-state automaton. If $L(\mathcal{A})$ is bounded then it is the union of the languages $w_{1} \sigma_{1}^{+} \cdots w_{d} \sigma_{d}^{+}$such that $\mathcal{A}$ contains an accepting run $q_{0} \stackrel{w_{1}}{\longrightarrow} q_{1} \stackrel{\sigma_{1}}{\longrightarrow} q_{1} \cdots q_{d-1} \stackrel{w_{d}}{\longrightarrow} q_{d} \stackrel{\sigma_{d}}{\longrightarrow} q_{d}$ with $d+\left|w_{1} \sigma_{1} \cdots w_{d} \sigma_{d}\right| \leq 6|Q|^{3}$.

Proof (sketch). The proof is obtained by first proving that $\left|w_{1} \sigma_{1}\right|, \ldots,\left|w_{d} \sigma_{d}\right|$ are bounded by $|Q|$. The lemma follows from the bound $d \leq|Q|^{2}+1$ with a pigeon-hole argument.

Now, consider an instance $(\mathcal{S}, \mathcal{A})$ of the context-freeness problem for flat counter systems. Recall that $L(\mathcal{A})$ is bounded. We derive from the previous Lemma that $\mathcal{T}(\mathcal{S}) \cap L(\mathcal{A})$ is not context-free if, and only if, there exists an accepting run $q_{0} \stackrel{w_{1}}{\longrightarrow} q_{1} \stackrel{\sigma_{1}}{\longrightarrow} q_{1} \cdots q_{d-1} \stackrel{w_{d}}{\longrightarrow} q_{d} \stackrel{\sigma_{d}}{\longrightarrow} q_{d}$ in $\mathcal{A}$ of polynomial length such that $\mathcal{T}(\mathcal{S}) \cap w_{1} \sigma_{1}^{+} \cdots w_{d} \sigma_{d}^{+}$is not context-free. Since non-context-freeness of $\mathcal{T}(\mathcal{S}) \cap w_{1} \sigma_{1}^{+} \cdots w_{d} \sigma_{d}^{+}$can be checked in NP, we obtain that the context-freeness problem for flat counter systems is in coNP. A matching lower bound is obtained by reduction from $3-\mathrm{SAT}$.

Lemma 6.6. The context-freeness problem for flat Petri nets ${ }^{8}$ is coNP-hard.

Proof (sketch). Given a 3-SAT formula $\varphi$, an instance $(\mathcal{S}, \mathcal{A})$ of the contextfreeness problem for flat Petri nets is computed in polynomial time in such a way that the language $\mathcal{T}(\mathcal{S}) \cap L(\mathcal{A})$ is non-empty, and in this case not context-free, if, and only if, the formula $\varphi$ is satisfiable. Since 3-SAT is NP-hard, the contextfreeness problem for flat Petri nets is coNP-hard.

We have shown the following theorem, which is the second main result of the paper.

Theorem 6.7. The context-freeness problem for flat counter systems is coNPcomplete, and remains so for flat Petri nets.

\footnotetext{
${ }^{8}$ The context-freeness problem for flat Petri nets is defined exactly as the contextfreeness problem for flat counter systems except that the input counter system is required to be a Petri net.
} 
Remark 6.8. Our setting requires a single initial configuration. Let us consider a variant without any specific initial configuration. An uninitialized counter system is a pair $\mathcal{S}=\langle c, \Theta\rangle$ where $c \in \mathbb{N}$ is a number of counters and $\Theta$ is a finite set of transitions. Its trace language is defined by $\mathcal{T}(\mathcal{S})=\bigcup_{\boldsymbol{x}_{\text {init }} \in \mathbb{N}^{c}} \mathcal{T}\left(\mathcal{S}, \boldsymbol{x}_{\text {init }}\right)$. The context-freeness problem for uninitialized flat counter systems is defined exactly as the context-freeness problem for flat counter systems except that it takes an uninitialized counter system as input. This problem can be shown to be decidable by adapting techniques developed in this paper. In fact, just observe that the set $\boldsymbol{N}=\left\{\left(n_{1}, \ldots, n_{d}\right) \in \mathbb{N}_{1}^{d} \mid w_{1} \sigma_{1}^{n_{1}} \cdots w_{d} \sigma_{d}^{n_{d}} \in \mathcal{T}(\mathcal{S})\right\}$ can be denoted by a Presburger formula $\varphi\left(n_{1}, \ldots, n_{d}\right)=\exists \boldsymbol{x}_{\text {init }} \boldsymbol{A}\left(\boldsymbol{x}_{\text {init }}, n_{1}, \ldots, n_{d}\right) \geq \boldsymbol{b}$ for a matrix $\boldsymbol{A}$ and a vector $\boldsymbol{b}$ that are both computable in polynomial time. Decidability of the context-freeness problem for uninitialized flat counter systems follows by quantifier elimination on $\varphi$. However, the complexity is open.

\section{Conclusions and Future Work}

The decidability of the stratifiability problem for semilinear sets was raised in $[13,14]$ almost fifty years ago, and is still open. Rephrased in terms of languages, this decision problem is equivalent to the question whether a given semilinear bounded language is context-free. In this paper, we have shown that the stratifiability problem for the subclass of integral polyhedra is coNP-complete. Building on this result, we have then established that the context-freeness problem for flat counter systems is coNP-complete, and remains coNP-hard for the subcase of flat Petri nets.

To solve the stratifiability problem for integral polyhedra, we have reduced it to the particular case of integral cones. While the latter is in coNP, its exact complexity is open.

\section{References}

1. Annichini, A., Bouajjani, A., Sighireanu, M.: TReX: A tool for reachability analysis of complex systems. In: Proc. CAV'01. LNCS, vol. 2102, pp. 368-372. Springer (2001)

2. Bardin, S., Finkel, A., Leroux, J., Petrucci, L.: Fast: acceleration from theory to practice. Int. J. Software Tools Technology Transfer (STTT) 10(5), 401-424 (2008)

3. Boigelot, B., Wolper, P.: Symbolic verification with periodic sets. In: Proc. CAV'94. LNCS, vol. 818, pp. 55-67. Springer (1994)

4. Bouajjani, A., Habermehl, P.: Symbolic reachability analysis of FIFO-channel systems with nonregular sets of configurations. Theor. Comput. Sci. 221(1-2), 211-250 (1999)

5. Bozga, M., Iosif, R., Konecný, F.: Fast acceleration of ultimately periodic relations. In: Proc. CAV'10. LNCS, vol. 6174, pp. 227-242. Springer (2010)

6. Cadilhac, M., Finkel, A., McKenzie, P.: Bounded parikh automata. Int. J. Found. Comput. Sci. 23(8), 1691-1710 (2012)

7. Chambart, P., Finkel, A., Schmitz, S.: Forward analysis and model checking for trace bounded WSTS. In: Proc. ICATPN'11. LNCS, vol. 6709, pp. 49-68. Springer (2011) 
8. Comon, H., Jurski, Y.: Multiple counters automata, safety analysis and Presburger arithmetic. In: Proc. CAV'98. LNCS, vol. 1427, pp. 268-279. Springer (1998)

9. Esparza, J., Ganty, P., Majumdar, R.: A perfect model for bounded verification. In: Proc. LICS'12. pp. 285-294. IEEE Computer Society (2012)

10. Finkel, A., Iyer, S.P., Sutre, G.: Well-abstracted transition systems: Application to FIFO automata. Information and Computation 181(1), 1-31 (2003)

11. Ginsburg, S., Spanier, E.H.: Bounded ALGOL-like languages. Trans. Amer. Math. Soc. 113, 333-368 (1964)

12. Ginsburg, S., Spanier, E.H.: Bounded regular sets. Proc. Amer. Math. Soc. 17(5), 1043-1049 (1966)

13. Ginsburg, S., Spanier, E.H.: Semigroups, Presburger formulas and languages. Pacific J. Math. 16(2), 285-296 (1966)

14. Ginsburg, S.: The Mathematical Theory of Context-Free Languages. McGraw-Hill (1966)

15. Hopcroft, J., Pansiot, J.J.: On the reachability problem for 5-dimensional vector addition systems. Theor. Comput. Sci. 8(2), 135-159 (1979)

16. Ibarra, O.H., Seki, S.: Characterizations of bounded semilinear languages by one-way and two-way deterministic machines. Int. J. Found. Comput. Sci. 23(6), 1291-1306 (2012)

17. Ibarra, O.H., Seki, S.: On the open problem of Ginsburg concerning semilinear sets and related problems. Theor. Comput. Sci. 501, 11-19 (2013)

18. Leroux, J., Penelle, V., Sutre, G.: On the context-freeness problem for vector addition systems. In: Proc. LICS'13. pp. 43-52. IEEE Computer Society (2013)

19. Leroux, J., Praveen, M., Sutre, G.: A relational trace logic for vector addition systems with application to context-freeness. In: Proc. CONCUR'13. LNCS, vol. 8052, pp. 137-151. Springer (2013)

20. Liu, L., Weiner, P.: A characterization of semilinear sets. J. Comput. Syst. Sci. 4(4), 299-307 (1970)

21. Schrijver, A.: Theory of Linear and Integer Programming. John Wiley and Sons, New York (1987)

22. Schwer, S.R.: The context-freeness of the languages associated with vector addition systems is decidable. Theor. Comput. Sci. 98(2), 199-247 (1992) 